\title{
What should referring doctors do if there is a delay in receiving COVID-19 test results and specialists require them for proper treatment of patients referred to them?
}

\begin{abstract}
To the Editor: The writer was recently asked by a specialist what should be done by referring doctors when there is a delay in receiving a patient's COVID-19 test results, and the patient needs to be referred to a specialist who needs the patient's COVID-19 status in order to treat the person properly. There appear to be no World Medical Association (WMA), Health Professions Council of South Africa (HPCSA) or other guidelines on how to deal with such situations.

In order to answer this question, it is necessary to discuss the types of COVID-19 tests available, and how long it takes for a patient's results to be received. It also requires a discussion of what referring doctors should do if the specialists to whom a patient is referred need to know the COVID-19 status of the patient for proper treatment, when: (i) it is not a medical emergency and the specialist treatment can be delayed; (ii) it is a medical emergency but the specialist treatment can be delayed for a short period of time; or (iii) it is a medical emergency that cannot be delayed even for a short period of time.
\end{abstract}

\section{Types of COVID-19 tests available and the time frames for their results}

COVID-19 viral tests in South Africa (SA) generally collect throat and/or nose swabs or other types of sample that are sent to a testing laboratory. The testing is done by both the SA National Health Laboratory Service (SANHLS) and private sector laboratories. The tests usually take about 48 hours, and the results are sent to both the patient and the patient's doctor. ${ }^{[1]}$ It is possible to get the test results sooner: for instance, the writer has personally undergone a COVID-19 test by a private laboratory that gave his doctor the results within 24 hours. The American Food and Drug Administration (FDA) has recently approved a new rapid test for COVID-19 that can yield results within 5 minutes, ${ }^{[2]}$ which will hopefully become available in SA sometime in the future. An antibody test cannot be used to determine if a person currently has COVID-19, as 'it may not be able to show if you have a current infection, because it can take 1 - 3 weeks after infection to make antibodies. ${ }^{[3]}$

Doctors should be aware, however, that even if a patient is asymptomatic, he or she may still transmit the virus..$^{[4]}$ Therefore, if the patient is asymptomatic but has been exposed to the risk of COVID19 infection, it is suggested that the referring doctors, specialists and support personnel should use personal protective equipment (PPE) to prevent them from being infected with COVID-19, in case the patient is able to transmit the virus.

Patients in the private sector requiring test results to be urgently provided for treatment purposes may now have their COVID-19 test paid for by their medical scheme - provided that they are referred for testing by a doctor. ${ }^{[5]}$ Patients in the public sector who require urgent results for treatment purposes have to rely on the relevant state health authority to make arrangements to obtain COVID-19 test results from a laboratory with a short turnaround time.

\author{
What should the referring doctor do if \\ it is not a medical emergency and the \\ specialist treatment can be delayed?
}

If there is a delay in receiving the COVID-19 test results (e.g. for a period of 10 days or 2 weeks), and the treatment may be delayed until after the expiry of this period, the referring doctor should be aware that results after such a long period may be based on a test done at a time when the patient was not infected, or may have been incubating the virus, which would not have been indicated by the test. ${ }^{[6]}$ Where the specialist needs to know the patient's COVID-19 status in order to treat the person properly, the referring doctor should arrange for a test to be done by a laboratory with a quick turnaround strategy, ${ }^{[2]}$ to ensure that the results are current - before the patient is referred to the specialist. Even then, if the patient tests negative, but has been exposed to the risk of COVID-19, the necessary PPE precautions should be taken by all the medical personnel concerned.

If the patient is in a public sector hospital, it may be necessary for the referring doctor to contact the relevant state health authorities, in order for arrangements to be made to deal with a laboratory that does COVID-19 testing in a short period of time. When doing so, the referring doctor should explain that at the time of the original test, the patient may not have had the virus, or was incubating it, and that it is essential for the patient's health that the specialist knows the current status of the patient, in order to treat the patient effectively without risking the person's health. It may be that if the health authority refuses to assist without reasonable justification, the official concerned, and the relevant health department, may be held legally liable for any injury or death resulting to the patient from a failure to provide for a current COVID-19 test. ${ }^{[7]}$

\section{What should the referring doctor do if it} is a medical emergency, but the specialist treatment can be delayed for a short period of time?

If the emergency medical treatment can be delayed for up to 24 hours, the referring doctor or specialist should arrange for the patient to be tested by a laboratory that undertakes to deliver the results to the patient and doctor within such a short period..$^{[2]}$ Again, if the patient is in a public sector hospital, the referring doctor should contact the relevant state health authorities to arrange for a test to be done by a private COVID-19 testing laboratory with a quick turnaround time, in order to protect the health of the patient. It should be made clear that to treat the patient properly, the specialist needs to know his or her current COVID-19 status. The referring doctor should also mention that if the patient was not infected, or still incubating the virus at the time of the first test, (s)he may have tested negative, and therefore a new test must be done to ascertain the patient's present status. 


\section{What should the referring doctor do if it is a medical emergency that cannot be delayed even for a short period of time?}

If the patient requires emergency medical treatment that cannot be delayed, both the SA Constitution ${ }^{[8]}$ (section 27(3)) and the National Health $\mathrm{Act}^{\left[{ }^{[9]}\right.}$ (section 5) require that nobody is refused emergency medical treatment, so the referring doctor should immediately refer the patient to a specialist, and advise the latter that the patient has been tested for COVID-19, but the results are not yet available. The specialist concerned will then have to make a judgement call on how to treat the patient without knowing their COVID-19 status. (S)he should try to assess clinically whether the patient may have COVID-19, and treat the patient accordingly. This should be done after having obtained the necessary informed consent or proxy consent, in terms of the National Health Act (section 7(1)), or without consent in terms of the Act, if there is no time to contact proxies, and the patient has not indicated in advance that (s)he does not wish to be treated in such circumstances (section 7(1)(e)).

The conduct of the specialist will be judged on the basis of what a reasonably competent specialist in the same field of practice would have done in similar circumstances. ${ }^{[10]}$ Provided that the specialist has acted in the same manner as a reasonably competent colleague would have done in the same situation, (s)he will not be legally liable if the patient is harmed or dies as a result of an incorrect judgement call that a reasonably competent colleague would also have made. ${ }^{[7]}$

This problem will be overcome in the longer term if the US FDAapproved 5-minute test ${ }^{[2]}$ becomes available in SA.

\section{Conclusion}

The above guidelines are suggested to assist doctors until such time as the WMA or HPCSA issue guidelines to deal with situations in which there is a delay in receiving a patient's COVID-19 test results, and the patient needs to be referred to a specialist who needs the patient's COVID-19 status to treat the person properly. If it is not a medical emergency, and the specialist treatment can be delayed, there is the problem that if at the time of the test 10 days or 2 weeks earlier, the patient's results were negative, as (s)he may not have had COVID-19, or was incubating the virus. Where the emergency treatment can be delayed for a short period of time (e.g. 24 hours), it may be possible to have a new test done at a laboratory with a very quick turnaround time.

However, if it is a medical emergency that cannot be delayed even for a short period of time, the specialist will have to make a judgement call on how to treat the patient, using a clinical diagnosis to determine whether or not the patient may have COVID-19. In such circumstances, provided the specialist acts as a reasonably competent colleague would have done in a similar situation, (s)he will not be legally liable for any harm caused to the patient or their family as a result of the wrong call. Hopefully, in the future, doctors will no longer have to face this dilemma if the US 5-minute test is approved for use in SA.

\section{J McQuoid-Mason}

Centre for Socio-Legal Studies, School of Law, University of KwaZuluNatal, Durban, South Africa

mcquoidm@ukzn.ac.za

S Afr J Bioethics Law 2020;13(1):5-6. https://doi.org/10.7196/SAJBL.2020. v13i1.734

1. Pathcare. Coronavirus information for patients. https://www.pathcare.co.za/drsportal/coronavirus/ (accessed 22 May 2020)

2. Wetsman N. A new COVID-19 test can return results in 5 minutes. https://www. theverge.com/2020/3/28/21197944/coronavirus-test-fast-doctors-office-abbottfda (accessed 25 May 2020).

3. Testing for COVID-19. https://www.cdc.gov/coronavirus/2019-ncov/symptomstesting/testing.html (accessed 25 May 2020).

4. Adejoro L. COVID-19: Asymptomatic patients are contagious, physicians warn https://www.msn.com/en-xl/news/other/covid-19-asymptomatic-patients-arecontagious-physicians-warn/ar-BB12v0oX?fullscreen=true\#timage $=1 \mathrm{~m}$ (accessed 23 May 2020).

5. BusinessTech. New coronavirus changes for South African medical aids. BusinessTech 11 May 2020. https://businesstech.co.za/news/finance/396519/ new-coronavirus-changes-for-south-african-medical-aids/ (accessed 23 May 2020).

6. CRTV. COVID-19: Why infected persons test negative and later positive. http:// www.crtv.cm/2020/03/covid-19-why-infected-persons-test-negative-and-laterpositive/ (accessed 25 May 2020)

7. McQuoid-Mason DJ. Public health officials and MECs should be held liable for harm caused to patients through incompetence, indifference, maladministration or negligence regarding the availability of hospital equipment. S Afr Med J 2016;106(7):681-683. https://doi.org/10.7196/SAMJ.2016.v106i7.10722

8. Constitution of the Republic of South Africa. 1996.

9. South Africa. National Health Act No. 61 of 2003.

10. Mitchell v Dixon 1914 AD 519 